\title{
Design Optimization of Linear Synchronous Motors for Overall Improvement of Thrust, Efficiency, Power Factor and Material Consumption
}

\author{
Sadegh Vaez-Zadeh ${ }^{\dagger}$ and Monir Sadat Hosseini* \\ $\dagger^{*}$ Center of Excellence on Applied Electromagnetics, School of Electrical and Computer Eng., The University of Tehran, \\ Tehran, Iran
}

\begin{abstract}
By having accurate knowledge of the magnetic field distribution and the thrust calculation in linear synchronous motors, assessing the performance and optimization of the motor design are possible. In this paper, after carrying out a performance analysis of a single-sided wound secondary linear synchronous motor by varying the motor design parameters in a layer model and a $d-q$ model, machine single- and multi-objective design optimizations are carried out to improve the thrust density of the motor based on the motor weight and the motor efficiency multiplied by its power factor by defining various objective functions including a flexible objective function. A genetic algorithm is employed to search for the optimal design. The results confirm that an overall improvement in the thrust mean, efficiency multiplied by the power factor, and thrust to the motor weight ratio are obtained. Several design conclusions are drawn from the motor analysis and the design optimization. Finally, a finite element analysis is employed to evaluate the effectiveness of the employed machine models and the proposed optimization method.
\end{abstract}

Key Words: Finite element analysis, Electromagnetic fields, Linear synchronous motors, Modelling, Optimization

\section{INTRODUCTION}

Linear synchronous motors (LSMs) enjoy high efficiency due to a lack of slip losses and a high magnetizing current. Also, the machine power factor can be controlled to higher values than a fixed power factor which is obtained with a comparable induction motor at the same output power and speed. A higher efficiency and power factor leads to a significant reduction in the inverter rating, resulting in a substantial cost saving. With an effective design optimization for LSMs, the benefits can be emphasized to varying degrees.

Many aspects of LSMs have been studied in the literature, including their modeling, analysis, design, control and optimization. Among these studies machine modeling plays a fundamental role since it is required for all other studies [1][4]. The design optimization of the motors is also important from a practical point of view since it reduces both the machine's primary and operating costs. It is carried out based on machine models given accurate knowledge of magnetic field distribution, thrust, efficiency, power factor, etc. Among the limited work on the design optimization of wound secondary linear synchronous motors (WSLSMs), the optimization of the secondary electromagnet shape has been investigated by using a finite element method (FEM) based model of the motor [5].

\footnotetext{
Manuscript received May 13, 2010; revised Oct. 12, 2010

$\dagger$ Corresponding Author: vaezs@ut.ac.ir

Tel: +98-21-6111-4916, Fax: +98-21-8877-8690, Tehran Univ.

* Dept. of Electrical and Computer Eng., Tehran University, Iran
}

However, a FEM based optimization is appropriate for the final stages of design optimization, is not time efficient for the iterative procedures in the pre-final stages. Alternatively, design optimizations based on analytical models are executed rapidly in comparison with FEM based optimizations. These optimization methods are necessary as the main tool to cope with the repeated runs of optimization procedures. However, analytical model based optimizations have focused heavily on permanent magnet linear synchronous motors (PMLSMs) rather than WSLSMs. Different objective functions and many design optimization variables are selected and studied for the optimization of such machines [6]-[11]. However, PMLSMs are mainly used in low power automation applications. They are still not considered for use in high power high speed applications like maglev.

In this paper a design optimization for WSLSMs based on an analytical model is presented. The thrust density of the motor based on its weight and the power factor multiplied by its efficiency are the two objective functions chosen for this research. These functions are chosen since $\eta \cos \varphi$ is an important machine characteristic for the inverter rating in motor design, and the thrust density is an essential factor in evaluating the motor performance. In fact, the thrust mean, weight, efficiency and power factor of a WRLSM are improved based on a machine layer model and a $d-q$ electrical model. The machine DC excitation pole dimensions, the pole pitch, the tooth width, the motor width, the primary current density, 


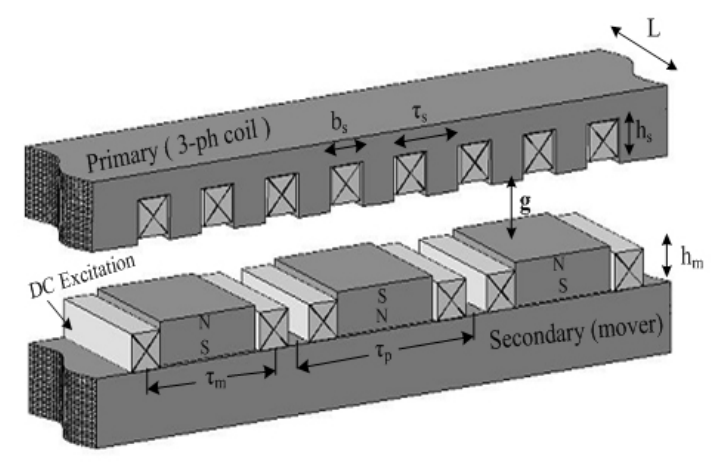

Fig. 1. Schematic view of a single-sided WSLSM.

the ratio of the $d$-axis to the $q$-axis component of the primary current and the yoke height are chosen as design variables. A flexible objective function is defined including the thrust to weight ratio, the efficiency and the power factor. A genetic algorithm (GA) optimization is then carried out to find out the best set of design variables. Finally, a finite element analysis is employed to verify the optimization results.

\section{MACHINE ModeL}

\section{A. Motor Topology}

Fig. 1 shows an upside down schematic view of a singlesided WSLSM with a long primary. The primary includes a three-phase iron-core winding which is extended along the motion path. The slot-openings of the windings total three slots. Three phases are supplied from several inverter stations which are placed along the path.

The moving part of the motor is a short secondary which consists of a back iron and DC excitation electromagnetic poles. The secondary is located above the primary with an air gap, $g$, and should experience a contactless motion along the path. The electromagnetic poles of the secondary consist of DC excited copper windings and iron cores which are connected to the back iron.

The parameters and dimensions of the motor are as follow: $\tau_{s}$ is the slot pitch, $b_{s}$ is the slot width, $h_{m}$ is the electromagnetic pole height, $\tau_{p}$ is the pole pitch, and $\tau_{m}$ is the electromagnet width which is the width of the iron core of a pole plus almost half of the DC coil width, $h_{s}$ is the slot height, and $L$ is the motor width along the $\mathrm{z}$ direction.

\section{B. Analytical Model}

In this subsection a method for developing a layer model for the motor presented in the previous subsection is briefly recalled [12]. All of the harmonics of the magnetic field produced by the DC-excited electromagnets are considered. The method is based on solving a Maxwell equations in several layers of the motor. The layer representing the DC excitation is modeled by an anisotropic layer with different permeability values along the $\mathrm{x}$ and $\mathrm{y}$ directions as follows, where the $\mathrm{x}$ axis is along the motor length and the $y$-axis is along the air gap [12]:

$$
\begin{aligned}
& \mu_{x m}=\mu_{0} \frac{\mu_{r}}{1+\left[\left(\tau_{p}-\tau_{m}\right) / \tau_{p}\right] \times\left(\mu_{r}-1\right)} \\
& \mu_{y m}=\mu_{0}\left[\left(\tau_{p}-\tau_{m}\right) / \tau_{p}+\mu_{r}\left(1-\left(\tau_{p}-\tau_{m}\right) / \tau_{p}\right)\right]
\end{aligned}
$$

and $\mu_{r}$ is the relative permeability of iron.

The Maxwell equations lead to Laplace and Poisson equations. It is known that the flux density in each layer can be achieved by the curl of the magnetic vector potential of the layer. As a result, the normal component of the flux density at $y=y_{0}$ in the air gap is given by [12]:

$B_{y}(x)=-\frac{\partial A}{\partial x}=-\sum_{n=1,3, \ldots}^{\infty} \frac{n \pi}{\tau_{p}}\left(C_{1} e^{\frac{n \pi y_{0}}{\tau_{p}}}+C_{2} e^{-\frac{n \pi y_{0}}{\tau_{p}}}\right) \cos \left(\frac{n \pi x}{\tau_{p}}\right)$

where $C_{1}-C_{2}$ can be found as:

$$
\left\{\begin{array}{l}
C_{1}=\frac{k(n)}{\left(1+e^{\frac{2 n \pi}{\tau_{p}} g_{e}}\right)-\frac{\sqrt{\mu_{x m} \mu_{y m}}}{\mu_{0}} \frac{\left(1+e^{-\sqrt{\frac{\mu_{x} m}{\mu_{y} m}} \frac{2 n \pi}{\tau_{p}} h_{m}}\right)\left(1-e^{\frac{2 n \pi}{\tau_{p}} g_{e}}\right)}{\left(1-e^{-\sqrt{\frac{\mu_{x m} m}{\mu_{y y}} \frac{2 n \pi}{\tau_{p}} h_{m}}}\right)}} \\
C_{2}=C_{1} e^{\frac{2 n \pi}{\tau_{p}} g_{e}}
\end{array}\right.
$$

where

$$
k(n)=\frac{\mu_{y m} J(n)}{n^{2} \pi^{2}} \tau_{p}^{2}
$$

and $J(n)$ is the maximum of the equivalent current density of the electromagnetic pole given by:

$$
J(n)=\frac{4}{\tau_{p}} \frac{N_{d c} I_{d c}}{h_{m}} \sin \left(\frac{\eta n \pi}{2}\right)
$$

where $\eta$ stands for the electromagnet width to pole pitch ratio.

\section{Machine Characteristics}

The flux density obtained in the previous subsection along with an electrical model of the machine is employed to calculate the motor characteristics including the developed thrust, the efficiency, the power factor, etc. A conventional $d-q$ electrical model of the machine in a synchronously rotating reference frame is used here. In this model, the flux distribution in the air gap - which is only produced by the electromagnetic poles - is assumed to be sinusoidal and the magnetic saturation is not considered. The motor thrust is then obtained as [7]:

$$
F_{x(a v)}=\frac{3}{2} \frac{\pi}{\tau_{p}}\left[\lambda_{D C-\text { excitation }}+\left(L_{d}-L_{q}\right) \times i_{d}\right] \times i_{q}
$$

In the motor considered in this paper $L_{d}$ is not equal to $L_{q}$, but for $i_{d}=0$ the second term of the thrust vanishes, so (6) is simplified to:

$$
\begin{aligned}
F_{x(a v)} & =\frac{3}{2} \frac{\pi}{\tau_{p}} \lambda_{D C-\text { excitation }} i_{q}=3 I_{p h} \times\left(B_{1 g} L N_{p h} k_{w 1}\right) \\
& =S_{1} B_{1 g} p \tau_{p} L
\end{aligned}
$$

where $B_{1 g}$ is the maximum of the fundamental harmonic of the field in the air gap which is obtained from (2), $I_{p h}$ is the maximum of the primary current, and $S_{1}$ is the maximum of the equivalent line current density of the stator. The direct axis current, $i_{d}$, is considered as a variable parameter.

In this kind of motor, the iron loss of the secondary is negligible. Therefore, the iron loss is due to the primary $\left(P_{i r o n}\right)$ 
which includes the eddy current, the hysteresis losses of the teeth and the stator yoke which are obtained as follows [13]:

$$
\left\{\begin{array}{l}
P_{\text {eddy }(\text { teeth })}=\frac{16 k_{\text {eddy }} \tau_{p} f^{2} B_{t(\max )}^{2}}{w_{t}} V_{\text {teeth }} \\
P_{\text {eddy }(\text { yoke })}=\frac{32 k_{\text {eddy }} \tau_{p} f^{2} B_{y(\max )}^{2}}{w_{t}} V_{\text {yoke }} \\
P_{\text {hyst }(\text { teeth })}=2 \pi f k_{\text {hyst }} B_{t(\max )}^{\beta} V_{\text {teeth }} \\
P_{\text {hyst }(\text { yoke })}=2 \pi f k_{\text {hyst }} B_{y(\max )}^{\beta} V_{\text {yoke }}
\end{array}\right.
$$

where $k_{\text {eddy }}$ is the coefficient of the eddy current losses which is a value between 0.04-0.07, according to the material characteristics of iron and its lamination, $k_{\text {hyst }}$ is the coefficient of the hysteresis losses which is a value between $40-50, \beta$ is a value between 1.8-2, $V_{\text {teeth }}$ is the total volume of the stator teeth and $V_{\text {yoke }}$ is the total volume of the stator yoke.

The other parts of the electrical loss are the copper losses of the primary and the secondary which are expressed as:

$$
\begin{aligned}
& P_{c u_{p}}=3 R_{p} I_{p h}^{2} \\
& P_{c u_{s}}=2 P R_{s} I_{f}^{2}
\end{aligned}
$$

where $R_{p}$ and $R_{s}$ are the resistances of the primary and the secondary windings respectively and $I_{f}$ is the DC current of the secondary. Also, there are additional losses $\left(P_{a d d}\right)$, including the mechanical loss and the stray loss. Therefore, the motor efficiency is given by:

$$
\eta=\frac{P_{\text {out }}}{P_{\text {out }}+P_{\text {iron }}+P_{c u}+P_{\text {add }}}
$$

where $P_{o u t}=F_{a v} . v_{s}$ and $v_{s}$ are the synchronous speed. Considering the $d$-axis current of the stator to be equal to zero, the power factor of the motor is obtained as follows:

$$
\cos \varphi=\frac{E_{f}+R_{p} I_{q}}{V_{t}}
$$

where $I_{q}$ is the stator current along the $q$-axis and $E_{f}$ and $V_{t}$ are the back EMF and the terminal voltage of the stator, respectively which are obtained as [14]:

$$
\begin{gathered}
E_{f}=4.44 f k_{w 1} \phi_{g} N_{p h} \\
V_{t}={\sqrt{\left(E_{f}-I_{d} X_{s d}\right)^{2}+\left(I_{q} X_{s q}\right)^{2}}}^{2}
\end{gathered}
$$

where $f$ is the frequency, $k_{w 1}$ is the armature winding coefficient $\Phi_{g}$ is the airgap magnetic flux, $N_{p h}$ is the number of the armature turns per phase and $X_{a d}$ and $X_{a q}$ are the d-axis and $\mathrm{q}$-axis armature reaction reactances respectively.

\section{Optimization Problem}

An optimization problem with $p$ objectives, $n$ variables, and $m$ constraints is formulated as

$$
\text { Maximize } f_{1}(x), f_{2}(x), \ldots, f_{p}(x) \quad x \in K
$$

where $x \in R^{n}$ and $f: R^{n} \rightarrow R$. Also, $K$ is a feasible set of solutions (15) which are described by

$$
K=\left\{x \in R^{n}: g_{i}(x) \leq 0, i=1,2, \ldots, p\right\}
$$

The constraints $g_{i}(x)$ limit the design variables. The design variables are chosen as the motor width, the electromagnet

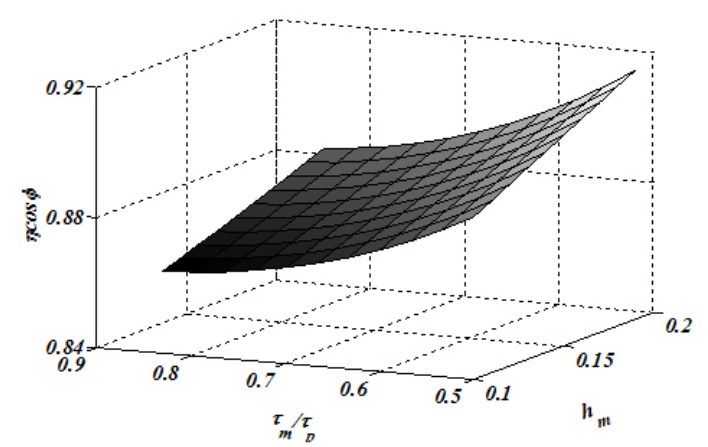

Fig. 2. Variations of efficiency multiplied by power factor with electromagnet dimensions.

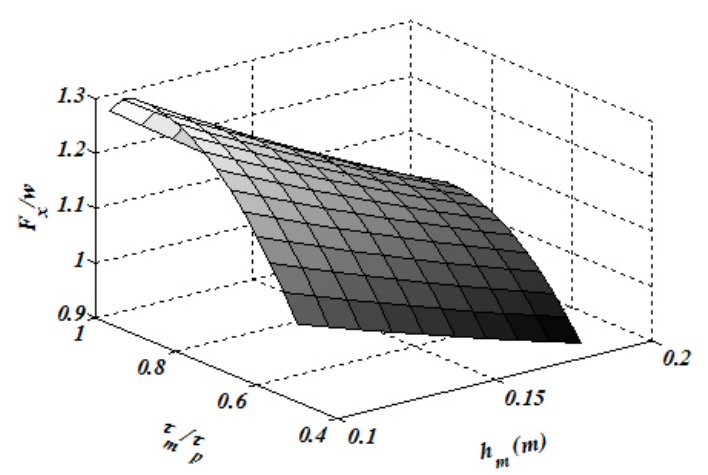

Fig. 3. Variations of thrust to weight ratio with electromagnet dimensions.

height, the electromagnet width to pole pitch ratio, the pole pitch, the yoke height, the primary current density $\left(J_{p}\right)$, the ratio of the $d$-axis to $q$-axis component of the primary current and the tooth width, based on their importance as influences on the optimization objectives as considered later in this section. The fixed variables are the slot pitch, the air gap, and the secondary windings current density according to their rather less important influences on the objectives.

The design objectives in this paper are to increase the motor developed thrust, the efficiency $(\eta)$ and the power factor $(\cos \varphi)$, and to reduce the motor weight. However, while copper is more expensive than iron, the iron lamination is also expensive. So to have a cost effective optimization the both copper weight and the iron weight are considered. The objectives improve the most important aspects of the motor performance and cost. For improving the efficiency and power factor we consider the objective function as $\eta \cos \varphi$, and for improving the motor thrust and reducing its weight the objective function of the thrust to weight ratio is selected. Since the length of the secondary is limited and the stator of the motor is as long as the motion path, the weight of the motor per meter $(w)$ instead of the total weight of the motor is considered as a design variable. The weight of the motor per unit of length is calculated as:

$$
\begin{aligned}
w & =\left(V_{c u-p} \times \rho_{c u}+V_{i-p} \times \rho_{i}+V_{c u-s} \times \rho_{c u}\right. \\
& \left.+V_{i-s} \times \rho_{i}\right) /\left(p \tau_{p}\right)
\end{aligned}
$$

where $V_{c u-p}, V_{c u-s}, V_{i-p}$, and $V_{i-s}$ are the primary and secondary copper volumes and the primary and secondary iron 


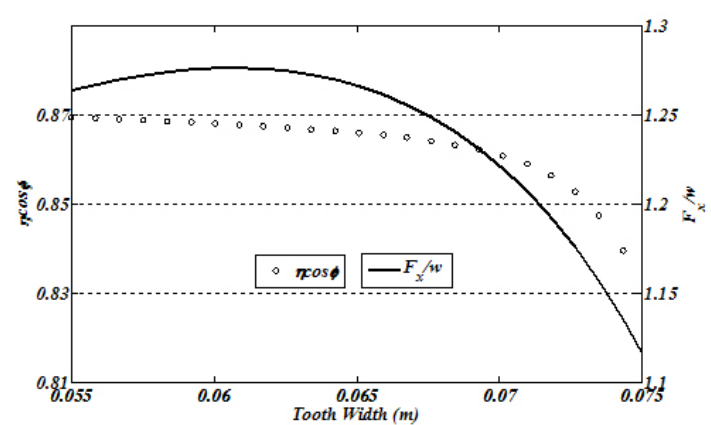

Fig. 4. Variations of thrust to weight ratio and $\eta \cos \varphi$ with the tooth width.

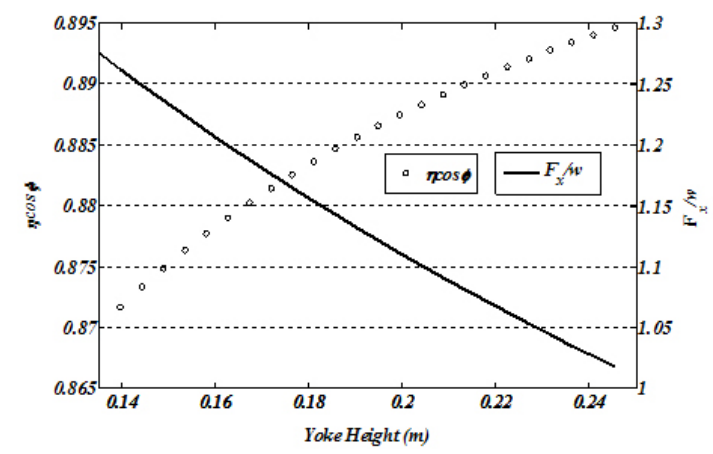

Fig. 5. Variations of thrust to weight ratio and $\eta \cos \varphi$ with the yoke height.

volumes, respectively and $\rho_{c u}$ and $\rho_{i}$ are the volume density of the copper and iron weights, respectively given as:

$$
\begin{aligned}
& \rho_{c u}=9.8 \times 10^{4}\left(\mathrm{~N} / \mathrm{m}^{3}\right) \\
& \rho_{i}=7.86 \times 10^{4}\left(\mathrm{~N} / \mathrm{m}^{3}\right) .
\end{aligned}
$$

An 18-pole WRLSM of the type depicted in Fig.1 is selected as the basis for optimization. Figs. 2-3 show the variations of objective functions $\eta \cos \varphi$ and the thrust to weight ratio in terms of the electromagnet dimensions. The objective function $\eta \cos \varphi$ decreases with an increase of the electromagnet width and increases with an increase of the electromagnet height (Fig. 2). However, the thrust to weight ratio varies in the opposite manner (Fig. 3). It can be concluded that the objectives do not have a simple common optimal point as far as electromagnet dimensions are concerned. In fact, meeting an objective may result in a deterioration of the other objectives.

Fig. 4 shows the variation of the thrust to weight ratio and $\eta \cos \varphi$ with the tooth width when the other motor variables are constant. These two objective functions are shown in Fig. 5 as a functions of the yoke height. As can be seen, an increase in the yoke height leads to an increase of $\eta \cos \varphi$ and a decrease in the thrust to weight ratio. Also, Fig. 6 shows the variation of the thrust to weight ratio and $\eta \cos \varphi$ with the pole pitch when the other motor variables are constant. As can be seen, an increase in the pole pitch leads to a decrease in the thrust to weight ratio and an increase of $\eta \cos \varphi$. Figs. 7-8 show the variations of the thrust to weight ratio and $\eta \cos \varphi$ with the primary current density and the motor width, respectively when the other motor variables are constant. As a result, both of the objectives increase with an increase in the motor width

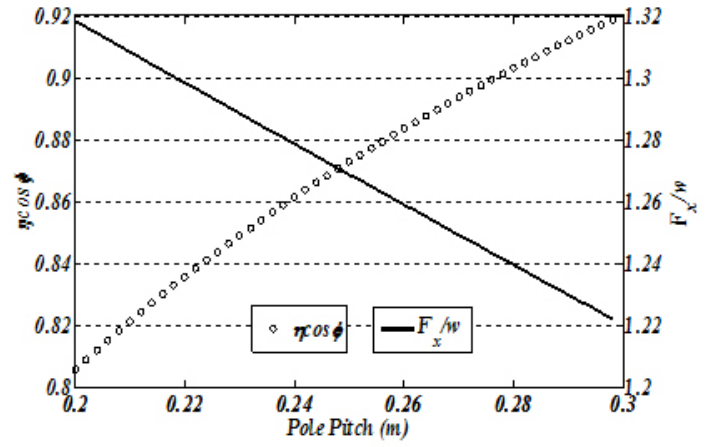

Fig. 6. Variations of thrust to weight ratio and $\eta \cos \varphi$ with the pole pitch.

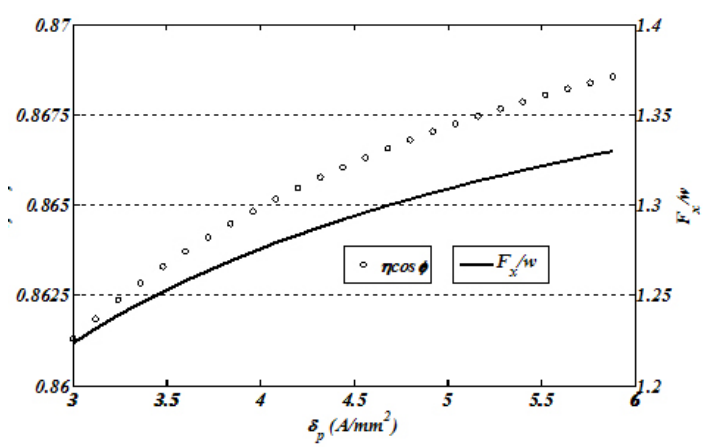

Fig. 7. Variations of thrust to weight ratio and $\eta \cos \varphi$ with primary current density.

and the primary current density. So, for maximizing them, the primary current density is set to its maximum value. In the case of motor width, it is necessary to consider that an unlimited increase in the motor width will lead to a large increase in the motor weight. Fig. 9 shows the variations of the thrust to weight ratio and $\eta \cos \varphi$ with the ratio of the $d$-axis to $q$ axis component of the primary current. As can be seen, the maximum of both objectives occurs at about $x=0.08$. So, for maximizing them, the ratio of the $d$-axis to $q$-axis primary currents is set to 0.08 .

Finally the results, presented by two-dimensional (2-D) and 3-D plots, confirm the necessity of an optimization regarding the motor thrust mean, the motor weight, the efficiency and the power factor. A wide variety of methods exists to solve this problem.

A widely used approach is based on a reduction of the objective functions of (15) to a single objective [15], [16]. In order to follow this approach, a new objective function is defined by combining the objectives with the cost coefficient or the cost powers [16]. The result is an objective function with the general form of:

$$
H=\left(F_{x} / w\right)^{a}(\eta \cos \varphi)^{b}
$$

is thus proposed for the optimization. The parameters $a$ and $b$ are chosen by the designer to determine the relative importance of each of the objective functions. A maximization of $H$ simultaneously fulfills all of the objectives of the optimization. Since both of the objective functions $(F / w$ and $\eta \cos \varphi)$ are positive and close to unity, their multiplication can be used as an objective function for the maximization of both. Such 


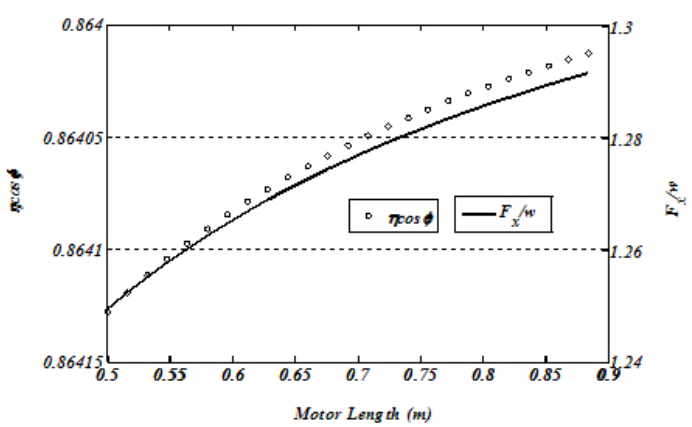

Fig. 8. Variations of thrust to weight ratio and $\eta \cos \varphi$ with the motor width.

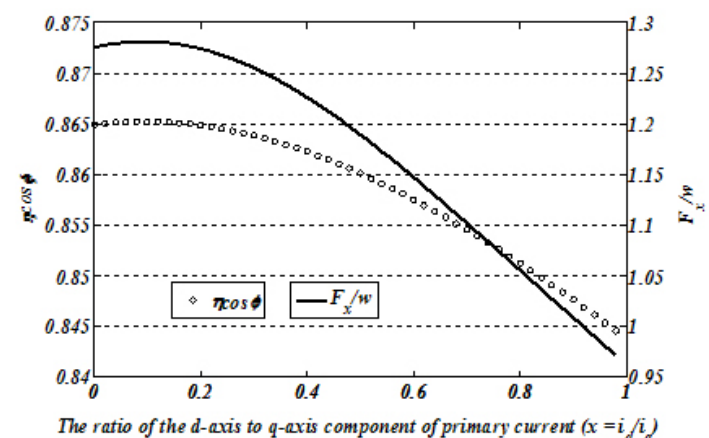

Fig. 9. Variations of thrust to weight ratio and $\eta \cos \varphi$ with the ratio of the $d$-axis to $q$-axis components of primary current.

an objective function provides a higher degree of freedom in selecting the appropriate motor parameters.

A number of constraints can also be taken into account during the optimization to prevent the possibility of reaching unrealistic optimization results. The motor width is limited by an upper bound to prevent low efficiency and by a lower bound to reduce leakage flux effect. The lower bound of the electromagnet height is dependent on the placing of DC excitation windings around it. The tooth width and the yoke height are limited by the lower bounds to prevent saturation. The primary current density bounds depend on the thermal constraints. Since the motor efficiency, the thrust and the power factor increase with an increase in motor size, limiting the upper bound of the motor weight is beneficial. Finally, the lower bound of the thrust is limited to the minimum required thrust. The limiting values of the design variables are listed in Table I.

TABLE I

DESIGN CONSTRAINTS

\begin{tabular}{|c|c|c|c|c|}
\hline Parameter & Unit & Symbol & Min & Max \\
\hline Pole Height & $m$ & $h_{m}$ & 0.1 & 0.2 \\
\hline $\begin{array}{l}\text { Electromagnet Width to } \\
\text { Pole Pitch Ratio }\end{array}$ & - & $\tau_{m} / \tau_{p}$ & 0.5 & 0.9 \\
\hline Tooth Width & $m$ & $w_{s}$ & 0.055 & 0.076 \\
\hline Primary Current Density & $\mathrm{A} / \mathrm{mm}^{2}$ & $J_{p}$ & 4 & 6 \\
\hline Yoke Height & $m$ & $h_{y}$ & 0.135 & 0.235 \\
\hline Weight & $k N / m$ & $w$ & - & 30 \\
\hline Pole Pitch & $m$ & $\tau_{p}$ & 0.2 & 0.3 \\
\hline $\begin{array}{l}\text { The Ratio of } d \text {-axis to } \\
q \text {-axis Currents }\end{array}$ & - & $x$ & 0 & 1 \\
\hline Thrust & $k N$ & $F_{x}$ & 30 & - \\
\hline Motor Width & $m$ & $L$ & 0.5 & 13.5 \\
\hline
\end{tabular}

TABLE II

Genetic Algorithm Parameters

\begin{tabular}{cc}
\hline Parameter & Max \\
\hline Probability of Tournament & 0.5 \\
Probability of Roulette wheel & 0.5 \\
Probability of crossover & 0.93 \\
Probability of mutation & 0.07 \\
Population size & 12 \\
Number of Generations & 40 \\
\hline
\end{tabular}

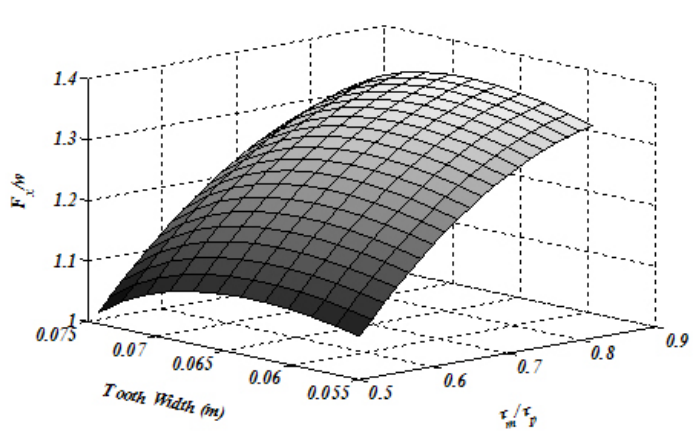

Fig. 10. Variations of thrust to weight ratio with electromagnet width and tooth width.

\section{Design Optimization}

Different design optimizations are carried out in this section depending on the selected objectives. The first optimization is aimed toward a maximization of the thrust to weight ratio. The next optimization is concerned with the maximization of the efficiency multiplied by the power factor. Finally, all the objectives are integrated into a single objective function. The general form of the objective function defined in (20) provides an opportunity to perform all of these optimizations according to the same procedure by choosing appropriate values for $a$ and $b$. A genetic algorithm (GA) is employed to find the optimal design in each optimization. However, it will be shown that for finding the optimal design in the thrust to weight ratio optimization a GA is not required.

\section{A. Genetic Algorithm}

A GA provides a random search technique to find the global optimal solution in a complex multidimensional search space. The algorithm consists of three basic operators, i.e. the selection, the crossover, and the mutation. First, an initial population is produced randomly. Then, the genetic operators are applied to the population to gradually improve their fitness. This procedure yields a new population in each iteration. In this paper, the Roulette wheel method and the Tournament method with equal probabilities are used for the selection of the parents' chromosomes [17]. The GA parameters used in this paper are shown in Table II.

\section{B. Maximization of the Thrust to Weight Ratio}

In this optimization, the objective function is defined as $F_{x} / w$. In this case the maximization of the thrust to weight ratio is aimed with an equal emphasis on thrust and weight. It can be concluded from Figs. 3-9 that for maximizing the thrust and minimizing the weight, most of the optimization problem take their boundary values. For example, the yoke, 


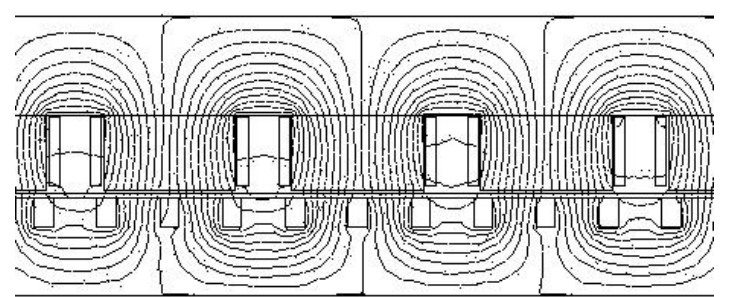

Fig. 11. Flux lines in the middle of the motor obtained by FEM.

TABLE III

DIMENSIONS AND CHARACTERISTICS OF OPTIMIZED MOTOR FOR INDIVIDUAL AND OVERALL OPTIMIZATIONS

\begin{tabular}{ccccc}
\hline Variable and \\
Parameter(Unit) & $\begin{array}{c}\text { Optimized } \\
\text { Motor1 }\end{array}$ & $\begin{array}{c}\text { Optimized } \\
\text { Motor2 }\end{array}$ & $\begin{array}{c}\text { Optimized } \\
\text { Motor3 }\end{array}$ & $\begin{array}{c}\text { Original } \\
\text { Motor }\end{array}$ \\
\hline Pole Height $(\mathrm{m})$ & 0.1 & 0.101 & 0.1043 & 0.1 \\
$\tau_{m} / \tau_{p}$ & 0.814 & 0.694 & 0.8139 & 0.72 \\
Pole Pitch $(\mathrm{m})$ & 0.2 & 0.3 & 0.2993 & 0.258 \\
Tooth Width $(\mathrm{m})$ & 0.0638 & 0.0615 & 0.0618 & 0.06 \\
$\delta\left(A / \mathrm{mm}^{2}\right)$ & 6 & 6 & 6 & 4 \\
The Ratio of $d$-axis & 0.08 & 0.08 & 0.08 & 0 \\
to $q$-axis Currents & & & 0.1389 & 0.135 \\
Yoke Height $(\mathrm{m})$ & 0.135 & 0.235 & 0.139 \\
Motor Width $(\mathrm{m})$ & 0.69 & 0.69 & 0.6513 & 0.69 \\
$\eta \cos \varphi$ & 0.8582 & 0.9311 & 0.9017 & 0.8641 \\
Losses $(100 \mathrm{~kW})$ & 4.5 & 2.32 & 3.81 & 3.77 \\
Thrust to weight ratio & 1.427 & 1.02 & 1.408 & 1.276 \\
Weight $(\mathrm{kN} / \mathrm{m})$ & 23.144 & 29.17 & 23.146 & 23.633 \\
Thrust $(\mathrm{kN})$ Analytical & 33.02 & 29.76 & 32.59 & 30.155 \\
FEM & 32.51 & 28.95 & 31.87 & 29.2 \\
\hline & & & &
\end{tabular}

the electromagnet heights and the pole pitch should be set to their minimum and the primary current density should be set to its maximum. Only the parameters of the tooth width and the electromagnet width to pole pitch ratio take values between their boundaries for optimization purposes. In this case, it is not necessary to use a GA for finding the optimal design. Since the number of variables is reduced to two variables, a 3-D figure is useful to give the optimal design. Fig. 10 shows the variations in the objective function of the thrust to weight ratio with the tooth width and the electromagnet width. The dimensions and characteristics of the optimized motor are shown in Table III as optimized motor1. In comparison with the original motor, in the optimized motor the ratio of thrust to weight increases from 1.276 to 1.427 , i.e. a $12 \%$ improvement. The motor losses are also calculated for both the optimized motor and the original motor using (8)-(10), where $v_{s}=111$ $\mathrm{m} / \mathrm{s}$ and $P_{\text {add }}=0.03 P_{\text {out }}$. This results in a $19 \%$ increase in the losses of the optimized motor. Also, the design provides a slightly lower $\eta \cos \varphi$. As a result, an overall optimization seems necessary.

\section{Maximizing of $\eta \cos \varphi$}

Optimizing this objective function using a GA results in a $7.8 \%$ increase in $\eta \cos \varphi$, i.e. from 0.8641 to 0.9311 . This results in a smaller inverter and therefore a lower cost. However, the objective function of the thrust to weight ratio is reduced by $20 \%$. The numerical parameters of the optimized motor are also listed in Table III as optimized motor2. As can be seen, the motor losses are reduced substantially but its weight is increased considerably. As a result, an overall optimization seems necessary.

\section{Overall Optimization}

The two optimizations presented above confirm the necessity for an overall optimization to achieve a high thrust, a low motor weight, and a high $\eta \cos \varphi$. Therefore, these objectives are simultaneously considered in the objective function of (20) by choosing appropriate values for $a$ and $b$. Although, in general the values of $a$ and $b$ depend on the designer's will and the requirements of the motor application; here more emphasis is placed on the maximization of $\eta \cos \varphi$ rather than the maximization of the thrust to weight ratio by choosing $a=1$ and $b=2$. This is because the results of the previous optimizations show that $\eta \cos \varphi$ is less sensitive to the optimization than the thrust to weight ratio. The results of the design optimization are listed in Table III as optimized motor3. Overall optimization leads to an improvement of $10.35 \%$ in the thrust to weight ratio, i.e., from 1.276 to 1.408 and an improvement of $4.35 \%$ in $\eta \cos \varphi$, i.e., from 0.8641 to 0.9017 . Also, the motor thrust increases considerably from $30.155 \mathrm{kN}$ to $32.59 \mathrm{kN}$ and the motor weight decreases from 23.633 $\mathrm{kN} / \mathrm{m}$ to $23.146 \mathrm{kN} / \mathrm{m}$. However, the motor losses increases from $3.77 * 100 \mathrm{~kW}$ to $3.91 * 100 \mathrm{~kW}$. Considering the desirable improvements in the motor thrust and $\eta \cos \varphi$, the decrease in motor weight and the slight increase in motor losses; the results show an overall improvement and are clearly better than the results of the previous two optimizations. Another advantage of the overall optimization is a reduction in the motor primary weight from $15.886 \mathrm{kN} / \mathrm{m}$ to $12.837 \mathrm{kN} / \mathrm{m}$ i.e. $19.2 \%$. Considering the motor topology which has a short secondary and a long primary, a reduction in the primary weight is more important than in the secondary weight as is achieved with this optimization.

\section{Design Evaluation}

The design optimizations in this work were carried out based on the analytical machine model presented in Section II. Therefore, validity of the design optimizations depends greatly on the accuracy of this model. However, the model is obtained through some simplifications such as ignoring the saturation and considering an unlimited motor length. Thus, it is necessary to evaluate the extent of the model accuracy. In this section, a 2-D finite element analysis is employed to evaluate the model. It is supposed that the motor is controlled with a current-controlled inverter. The forces are then calculated using the local virtual work method. Numerical and graphical results are obtained. Fig. 11 shows a graphical representation of the flux lines in the WRLSM. The thrusts for different designs are obtained by the FEM and are compared with those obtained by the analytical model as in Table III. It is seen that the results of the analytical model are very close to the results of the FEM. The maximum error in the case of the thrust calculation is less than $4 \%$, which is reasonable. This proves the validity of the proposed analytical model based design optimization.

\section{CONCLUSions}

Different design optimizations are performed on a WRLSM to achieve a high developed thrust, a reduced motor weight, 
and a high $\eta \cos \varphi$. A layer model and a $d-q$ model of the machine are used in defining the optimization problem in each case. Low efficiency is avoided by limiting the motor width by an upper bound. The machine's DC excitation pole dimensions, the pole pitch, the tooth width, the motor width, the primary current density, the ratio of the $d$-axis to $q$-axis component of the primary current and the yoke height are found to be appropriate design variables due to their rather important influence on the objective functions.

A genetic algorithm is employed to search for the optimal design variables. By considering a lower bound constraint for the motor developed thrust, the thrust to weight ratio and $\eta \cos \varphi$ are optimized independently to $12 \%$ and $7.8 \%$ of those of the original motor, respectively. These two optimizations confirm the necessity of an overall design optimization taking into account both the thrust to weight ratio and $\eta \cos \varphi$ simultaneously. The results of such an optimization show that it can increase both the thrust to weight ratio and $\eta \cos \varphi$. Although, a slight increase in the motor losses can be observed, the results show an overall improvement in the objectives and they are clearly better than the results of the previous two optimizations.

Finally a finite element analysis confirms both the validity of the analytical model used and the performed design optimizations. However, taking into account the specific phenomena of the machine such as the end effects and the rather large air gap, an experimental evaluation of the machine would be useful for further confirmation of the optimization and this is recommended as a future step.

\section{REFERENCES}

[1] H. W. Cho, H. K. Sung, S. Y. Sung, D. J. You, and S. M. Jang, "Design and characteristic analysis on the short-stator linear synchronous motor for high-speed maglev propulsion," IEEE Trans. Magn., Vol. 44, No. 11, pp. 4369-4372, Nov. 2008.

[2] Y. M. Chen, S. Y. Fan, and W. S. Lu, "Performance analysis of linear permanent-magnet synchronous motors with finite-element analysis,' IEEE Trans. Magn., Vol. 44, No. 3, pp. 377-385, Mar. 2008.

[3] B. Tomczuk, G. Schröder, and A. Waindok, "Finite-element analysis of the magnetic field and electromechanical parameters calculation for a slotted permanent-magnet tubular linear motor," IEEE Trans. Magn., Vol. 43, No. 7, pp. 3229-3236, Jul. 2007.

[4] O. Danielsson and M. Leijon, "Flux distribution in linear permanentmagnet synchronous machines including longitudinal end effects," IEEE Trans. Magn., Vol. 43, No. 7, pp. 3197-3201, Jul. 2007.

[5] D. Y. Jeon, D. Kim, and S.Y. Hahn, "Optimum design of linear synchronous motor using evolution strategy combined with stochastic FEM," IEEE Trans. Magn., Vol. 35, No. 3, pp. 1734-1737, May 1999.

[6] S. I. Kim, J. P. Hong, Y. K. Kim, H. Nam, and H. I. Cho, "Optimal design of slotless-type PMLSM considering multiple responses by response surface methodology," IEEE Trans. Magn., Vol. 42, No. 4, pp. 12191222, Apr. 2006.

[7] S. Vaez-Zadeh and A. Hassanpour, "Multiobjective design optimization of air-core linear permanent magnet synchronous motors for improved thrust and low magnet consumption," IEEE Trans. Magn., Vol. 42, No. 3, pp. 446-452, Mar. 2006.

[8] D. Y. Lee, C. G. Jung, K. J. Yoon, and G. T. Kim, "A study on the efficiency optimum design of a permanent magnet type linear synchronous motor," IEEE Trans. Magn., Vol. 41, No. 5, pp. 18601863, May 2005.
[9] D. Y. Lee and G. T. Kim, "Design of thrust ripple minimization by equivalent magnetizing current considering slot effect," IEEE Trans. Magn., Vol. 42, No. 4, pp. 1367-1370, Apr. 2006.

[10] Y. W. Zhu, D. H. Koo, and Y. H. Cho, "Detent force minimization of permanent magnet linear synchronous motor by means of two different methods," IEEE Trans. Magn., Vol. 44, No. 11, pp. 4345-4348, Nov. 2008.

[11] J. Wang and D. Howe, "Design optimization of radially magnetized, iron-cored, tubular permanent-magnet Mmachines and drive systems," IEEE Trans. Magn., Vol. 40, No. 5, pp. 3262-3277, Sep. 2004.

[12] M. S. Hosseini and S. Vaez-Zadeh, "Modeling and analysis of linear synchronous motors in high speed maglev vehicles," IEEE Trans. on Magnetics, Vol. 46, No. 7, pp. 2656-2664, Jul. 2010.

[13] S. Meier, Theoretical design of Surface Mounted permanent magnet motors with Field-weakening capability, MSc thesis, Royal Inst. Tech., Stockholm, Sweden, 2001/2002.

[14] J. G. Gieras and Z. J. Piech, Linear Synchronous Motors. Boca Raton, FL: CRC, chap. 3, 2000.

[15] G. Liuzzi, S. Lucidi, F. Parasiliti, and M. Villani, "Multiobjective optimization techniques for the design of induction motors," IEEE Trans. Magn., Vol. 39, No. 3, pp. 1261-1264, May 2003.

[16] S. Vaez-Zadeh and A. R. Ghasemi, "Design optimization of permanent magnet synchronous motors for high torque capability and low magnet volume," Elect. Power Syst. Res., Vol. 74, pp. 307-313, Mar. 2005.

[17] N. Bianchi and S. Bolognani, "Design optimization of electric motors by genetic algorithm," IEE Proc. - Electr. Power Appl., Vol. 145, No. 5, pp. 475-483, Sep. 1998

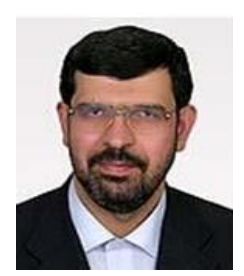

Sadegh Vaez-Zadeh received his B.S. from the Iran University of Science and Technology, Tehran, Iran, in 1985 and his M.S. and Ph.D. from Queen's University, Kingston, ON, Canada, in 1993 and 1997, respectively, all in Electrical Engineering. He was with several research and educational institutions in different positions before joining the University of Tehran in 1997 as an Assistant Professor. He became an Associate Professor in 2001 and a Full Professor in 2005. He served the university as the Head of the Power Division from 1998 to 2000 and is currently the Director of the Advanced Motion Systems Research Laboratory which he founded in 1998 and the Director of the Electrical Engineering Laboratory since 1998. He has been active in IEEE sponsored conferences as a member of technical and steering committees, session chair, etc. His research interests include advanced rotary and linear electric machines and drives, motion control, magnetic levitation, electric and hybrid vehicles and power system stability and control. He has published over 150 technical papers in these areas. Professor Vaez-Zadeh is a member of the IEEE PES Motor Sub-Committee and the Power System Stability Control Sub-Committee. $\mathrm{He}$ is an Editor of the IEEE Transactions on Energy Conversion and a Founding Member of the editorial board of the Iranian Journal of Electrical and Computer Engineering. He is also a Member of the editorial board of the Journal of Faculty of Engineering, which is the oldest Engineering Journal in the Middle East and a Member of the editorial board of the newly established International Journal on Power System Optimization. He has received a number of awards domestically including a Best Paper award from Iran Ministry of Science, Research and Technology in 2001 and a Best Research award from the University of Tehran in 2004.

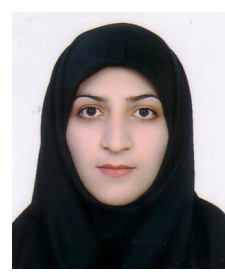

Monir Sadat Hosseini was born in Karaj, Tehran, Iran, in 1983. She received her B.S. in Electrical Engineering from the University of Tehran, Tehran, Iran, in 2005, where she also received her M.S. in Power Engineering in 2009. Her research interests include the design, modeling, and control of electrical machines. 